\title{
Lower Parathyroid Hormone-Related Protein Content of Tracheal Aspirates in Very Low Birth Weight Infants Who Develop Bronchopulmonary Dysplasia
}

\author{
VIRENDER K. REHAN AND JOHN S. TORDAY \\ Department of Pediatrics [V.K.R., J.S.T], Department of Obstetrics and Gynecology [J.S.T.], Harbor-UCLA Medical Center, Los Angeles \\ Biomedical Research Institute at Harbor-UCLA, David Geffen School of Medicine at UCLA, Torrance, California, 90502
}

\begin{abstract}
Since parathyroid hormone-related protein (PTHrP) secreted by pulmonary alveolar type II cells is a key physiologic paracrine factor in maintaining alveolar homeostasis, we hypothesized that its levels in the tracheal aspirates (TA) of ventilated very low birth weight infants (VLBWI) would correlate with the development of bronchopulmonary dysplasia (BPD). Therefore, we examined whether TA PTHrP content during the first week of life correlates with the later development of BPD. Forty VLBWI [birth weight, $943 \pm 302 \mathrm{~g}$ (mean $\pm \mathrm{SD}$ ); gestational age, $27 \pm 2 \mathrm{wk} ; 21$ males and 19 females], who were ventilated for respiratory distress syndrome, were studied. The TA were collected once daily until the infants were extubated and immediately frozen at $-70^{\circ} \mathrm{C}$ for subsequent assays for PTHrP and matrix metalloproteinase-8 (MMP-8), a previously described, nonspecific TA biomarker for BPD. The levels of these proteins were correlated with the later development of BPD. PTHrP in the TA during the first week of life was significantly lower in those infants who developed BPD (12/40) than among those who did not (28/40). The PTHrP levels also correlated with the duration of mechanical ventilation needed in these infants. In contrast, MMP-8 levels did not correlate with BPD. We conclude that lower TA PTHrP content during the first week of life in ventilated VLBWI inversely correlates with prolonged ventilation and the later development of BPD. (Pediatr Res 60: 216-220, 2006)
\end{abstract}

$\mathrm{D}^{\mathrm{e}}$ espite tremendous improvements in preterm neonatal care, BPD remains a common sequela in preterm infants (1), and, in fact, may be on the rise due to the increased overall survival of VLBWI (2). Its pathogenesis remains incompletely understood and there are neither reliable early predictors nor any effective treatment options. Abnormal airway development and an arrest in alveolarization (3) are the histologic hallmarks of the "new BPD," which is seen characteristically in extremely low birthweight infants. Mechanical ventilation (4), oxygen exposure (5), and inflammation (6) of the premature lung are important contributing factors, which are all known to disrupt normal pulmonary development during the

Received October 25, 2005; accepted March 25, 2006.

Correspondence: Virender K. Rehan, M.D., Department of Pediatrics, Los Angeles Biomedical Research Institute at Harbor UCLA Medical Center, David Geffen School of Medicine at UCLA, 1124 West Carson Street, Torrance, CA 90502; e-mail: vrehan@labiomed.org

This study was supported by grants from the National Institutes of Health (HL55268 to JST and VKR, HL075405 to VKR and JST, and 3 MO1 RR00425 to GCRC), the American Heart Association (10868-02 to VKR), and Philip Morris Inc. (11108-02 to VKR).

DOI: 10.1203/01.pdr.0000228328.93773.27 critical period of lung growth and development, resulting in arrested alveolarization and BPD.

Pulmonary epithelial-mesenchymal interactions, through the secretion of various autocrine and paracrine factors, are essential for normal alveolar growth and development (7). $\mathrm{PTHrP}$ is an important paracrine factor that plays an essential role in pulmonary epithelial and mesenchymal cytodifferentiation (8) through epithelial-mesenchymal communications in the developing lung alveolus $(9-11)$. We have recently shown that alveolar overdistension (12) and/or exposure to hyperoxia (13) down-regulate alveolar PTHrP signaling. Because of both its essential role in the developing alveolus and its downregulation by factors that lead to $\mathrm{BPD}$, we hypothesized that PTHrP levels in the TA fluid of ventilated VLBWI would correlate with the development of BPD.

The aim of this study was to examine whether the TA PTHrP content during the first week of life would correlate with the development of BPD. We also wanted to compare TA PTHrP with TA matrix metalloproteinase (MMP)-8 levels, a previously described, nonspecific tracheal aspirate biomarker for BPD in preterm infants $(14,15)$.

\section{METHODS}

Forty VLBWI (birth weight $\leq 1500 \mathrm{~g}$ ) admitted consecutively to the neonatal intensive care unit of Harbor-UCLA Medical Center between January 1999 and October 2002, who were ventilated for respiratory distress syndrome, were prospectively enrolled in this study. Infants with major anomalies were not included in this study. Informed consent was obtained from the parents and the study was approved by the ethics committee at the Los Angeles Biomedical Research Institute at Harbor-UCLA Medical Center.

Tracheal aspirate. Using a standard protocol, tracheal aspirates were collected once daily until the infants were extubated. One milliliter per kilogram of sterile saline was instilled through a $5 \mathrm{~F}$ catheter inserted into the trachea via the endotracheal tube and immediately aspirated back into a suction trap. The collected sample was immediately frozen at $-70^{\circ} \mathrm{C}$ for subsequent analysis after adding a mixture of the protease inhibitors leupeptin, pepstatin, and aprotinin (Sigma Chemical Co., St. Louis, MO) at $1 \mu \mathrm{g} / \mathrm{mL}$

Abbreviations: AIF, alveolar interstitial fibroblasts; ATII, alveolar type II; BPD, bronchopulmonary dysplasia; MMP, matrix metalloproteinase; PTHrP, parathyroid hormone-related protein; TA, tracheal aspirate; VLBWI, very low birth weight infants 
for each. For the purposes of subsequent analysis, for each patient, samples collected on all days of each week were pooled. Clinical data on demographics, respiratory, and nonrespiratory morbidity and mortality were collected prospectively.

Assays for PTHrP And MMP-8. Pooled TA samples were centrifuged (1500 rpm at $4{ }^{\circ} \mathrm{C}$ for $10 \mathrm{~min}$ ) and the supernatants were then processed to quantitate the levels of MMP-8 and PTHrP, using ELISA (Amersham Pharmacia Biotech, Piscataway, NJ) and Immunoradiometric (Quest Diagnostics, San Juan Capistrano, CA) assays, respectively, according to the manufacturer's instructions. These assays are highly reproducible, with assay sensitivities of $0.3 \mathrm{pM} / \mathrm{L}$ for PTHrP and $0.03 \mathrm{ng} / \mathrm{mL}$ for MMP- 8 assay. The PTHrP and MMP-8 assays were run without knowledge of the clinical course of the recruited infants. The protein content of the TA was determined by the Bradford dye-binding method $(15,16)$. Tracheal aspirate PTHrP and MMP-8 levels were correlated with the presence or absence of BPD, as defined by the need for oxygen at $36 \mathrm{wk}$ postconceptional age to maintain an oxygen saturation of greater than $90 \%$ by pulse oximeter, along with the presence of radiologic changes on chest radiographs consistent with BPD, as determined by a single investigator (VKR).

Respiratory distress syndrome was diagnosed in the presence of clinical respiratory distress along with typical radiologic appearance of hyaline membrane disease, i.e. lung field granularity, small lung volumes, and air bronchograms. Air leak was diagnosed in the presence of a pneumothorax or a pneumomediastinum. The mean $\mathrm{FiO}_{2}$ and mean airway pressure were calculated as the means of the highest $\mathrm{FiO}_{2}$ and mean airway pressure recorded for each day for the duration of the oxygen therapy and the ventilatory support, respectively.

Statistical analysis. Population data are expressed as mean \pm SD and were analyzed using the unpaired $t$ test or Mann Whitney $U$ test, as appropriate. PTHrP and MMP-8 data are expressed as mean \pm SEM. ANOVA or $t$ tests were used to compare means. A receiver operating characteristic (ROC) curve was constructed that displays the sensitivity and specificity of PTHrP as a diagnostic predictor of BPD according to varying potential cutoff PTHrP values. The optimal cutoff was selected as that PTHrP value that maximizes sensitivity plus specificity. The log-rank test was used to compare, between subgroups of infants above or below the optimal diagnostic PTHrP level, Kaplan-Meier curves that express for each subgroup the probability of remaining on ventilation over time by cumulating infants weaned off ventilatory support. Logistic regression was used to estimate the relative risks of BPD for prespecified known risk factors and for PTHrP, with simultaneous mutual adjustment of these risk factors for each other, specified a priori and regardless of individual significance in this data. A $p$ value of $<0.05$ was considered significant. Based on TA PTHrP values from a baboon model and our preliminary data in humans, we calculated that a sample size of 10 infants in each group would be sufficient to reject or accept the null hypothesis that mean first week TA PTHrP levels would be no different in infants who do or do not develop BPD (power of $90 \%$ to detect a mean difference of $25 \%$ in PTHrP levels, and a significance level of 5\%, two-tailed test).

\section{RESULTS}

Forty VLBWI [birth weight, $943 \pm 302 \mathrm{~g}$ (mean $\pm \mathrm{SD}$ ); gestational age, $27 \pm 2$ wk; 21 males and 19 females, 10 delivered vaginally and 30 through cesarean section] were recruited. The median Apgar scores were 4 (range, 1-8,) and 7 (range, 4-9) at 1 and $5 \mathrm{~min}$, respectively. All infants received at least one dose of surfactant (Infasurf, Forest Pharmaceuticals, Inc., St. Louis, MO) at a dosage of $3 \mathrm{~mL} / \mathrm{kg}$ (phospholipids $105 \mathrm{mg} / \mathrm{kg}$ ). Overall, 12 out of 40 infants (30\%) subsequently developed BPD as defined by the previously described criteria. All mothers [32/40 (80\%)], except for four from each group (BPD and non-BPD), had received at least one dose of antenatal steroid before delivery. Two infants, one each from the BPD and non-BPD groups, died.

Figure 1 shows TA PTHrP levels during the first week in infants who later did or did not develop BPD. Infants who developed BPD had 3-fold lower PTHrP levels during the first week versus patients who did not develop BPD (Fig. 1). Further, first-week PTHrP levels also correlated with the duration of ventilation needed. Infants requiring only $1 \mathrm{wk}$ of

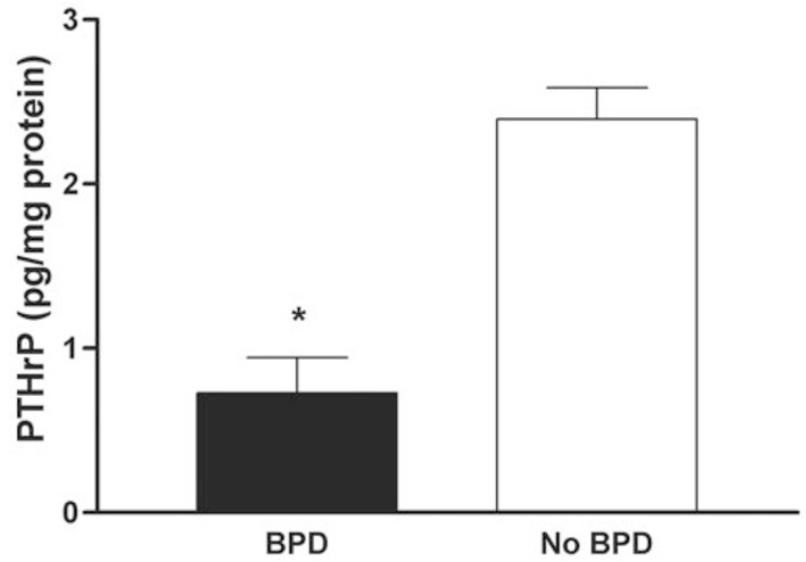

Figure 1. Infants who later went on to develop BPD $(n=12)$ had significantly lower tracheal aspirate PTHrP levels during the first week vs patients who did not develop BPD $(n=28)\left({ }^{*} p<0.001\right.$, BPD $v s$ no BPD). Values are mean \pm SEM

ventilation had significantly higher wk 1 PTHrP levels than infants who required 2 or 3 wk of ventilation, $p<0.0001$ for linear trend over weeks (Fig. 2). In contrast to PTHrP levels, first-week TA MMP-8 levels were not different in the infants who later did or did not develop BPD (Fig. 3). To determine the value of using various cutoff levels for the TA PTHrP for predicting BPD, a ROC analysis was performed (Fig. 4). A PTHrP level of $\leq 1.32 \mathrm{pg} / \mathrm{mg}$ protein predicts the later development of BPD maximally [84.6\% correct classifications (true positives + true negatives), most upper-left point on ROC curve (Fig. 4)], with a sensitivity $=76.9 \%$ and specificity $=$ $88.5 \%$. Other cutoffs that put greater emphasis on either sensitivity or specificity are almost as good; cutoffs between 0.43 and $1.89 \mathrm{pg} / \mathrm{mg}$ protein provide overall percentage correct classifications of at least $76.9 \%$. We next examined whether this optimal cutoff value of TA PTHrP $(1.32 \mathrm{pg} / \mathrm{mg}$ protein) discriminated infants according to the duration of ventilation needed. To examine this, using TA PTHrP level of

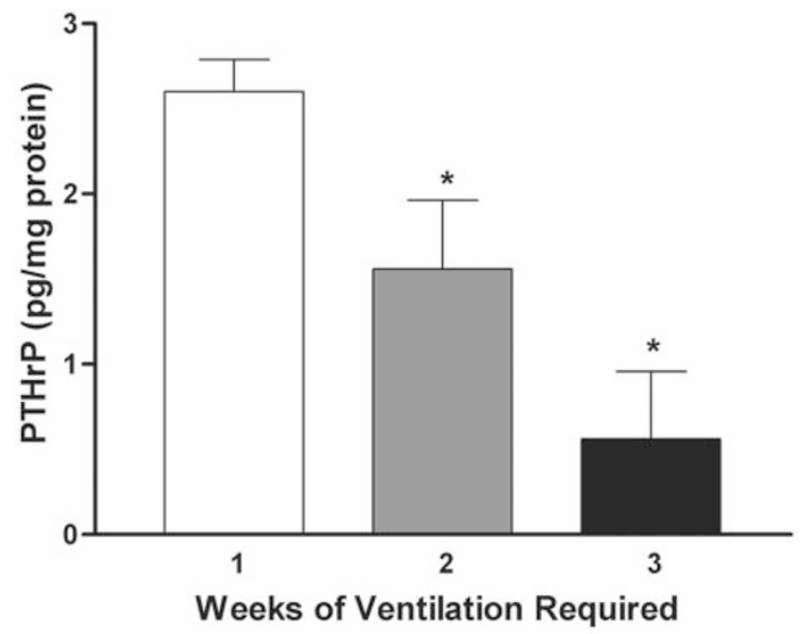

Figure 2. Infants with significantly higher levels of PTHrP levels during the first week required only $1 \mathrm{wk}(n=22)$ of ventilation $v s$ infants with lower levels of PTHrP who required $2(n=8)$ or $3(n=10)$ wk of ventilation $(* p$ $<0.05$, weeks of ventilation required $v s 1 \mathrm{wk}$ of ventilation required). Values are mean \pm SEM 


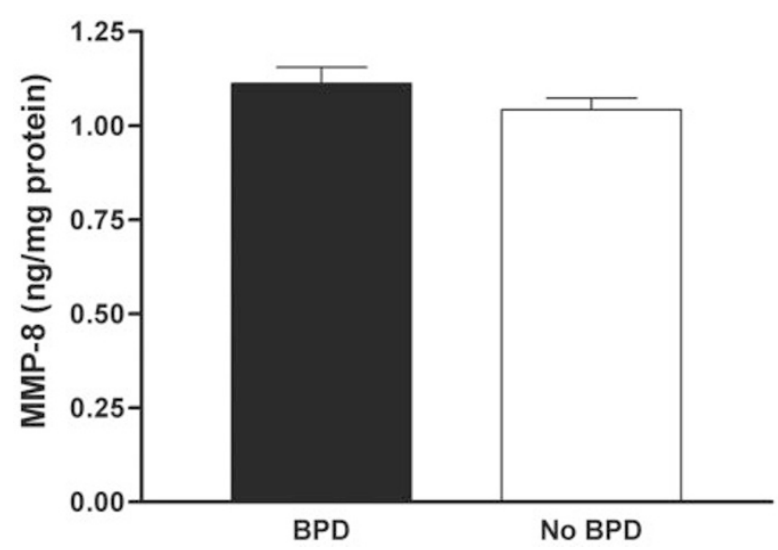

Figure 3. Tracheal aspirate matrix metalloproteinase-8 (MMP-8) levels during the first week were not different in infants who subsequently did $(n=12)$ or did not develop BPD $(n=28)$. Values are mean \pm SEM.

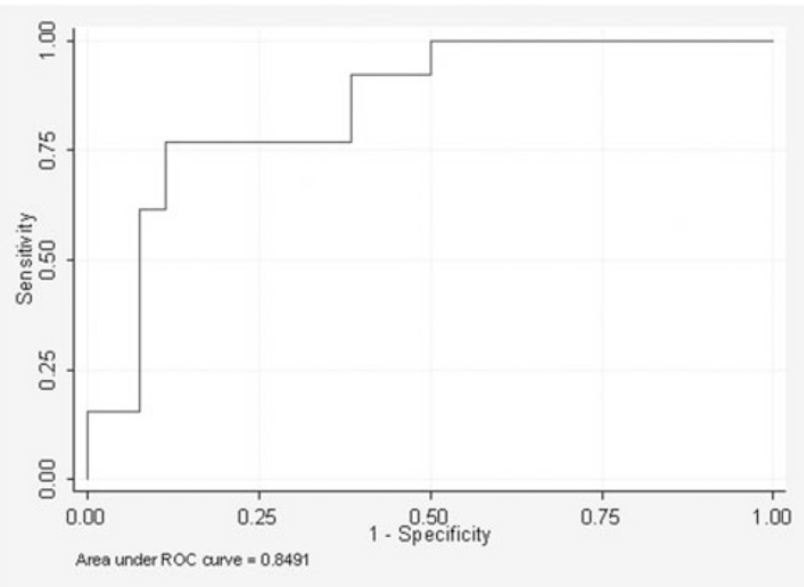

Figure 4. ROC curve for predicting the later development of BPD based on first week TA PTHrP level. A value of $\leq 1.32 \mathrm{pg} / \mathrm{mg}$ protein predicts the later development of BPD maximally ( $84.6 \%$ true positives + true negatives, most upper-left point on ROC curve), with a sensitivity $=76.9 \%$ and specificity $=$ $88.5 \%$.

$1.32 \mathrm{pg} / \mathrm{mg}$ protein as the cutoff, we constructed Kaplan-Meier curves to compare the duration of ventilation needed between the two groups, i.e. less than or equal to and more than 1.32 $\mathrm{pg} / \mathrm{mg}$ protein TA PTHrP level (Fig. 5). Infants with TA PTHrP levels $>1.32 \mathrm{pg} / \mathrm{mg}$ protein were off ventilatory support significantly earlier $(p<0.0001)$. As expected, the mean airway pressure and mean fractional inspired oxygen concentration required for the group with TA PTHrP values $\leq 1.32$ $\mathrm{pg} / \mathrm{mg}$ protein were significantly higher compared with the group with TA PTHrP values $>1.32 \mathrm{pg} / \mathrm{mg}$ protein (Fig. 6). However, mean GA, mean BW, and sex distribution between infants with low and high TA PTHrP values were not significantly different (Table 1). Lastly, to determine how PTHrP levels compare with the other known predictors of BPD, such as birth weight $(<1000 \mathrm{~g})$, GA $(<28 \mathrm{wk})$, and sex (male gender), we performed multivariate logistic regression analysis for these four variables in predicting the development of BPD (Table 2). Of these variables, a PTHrP level of $\leq 1.32$ $\mathrm{pg} / \mathrm{mg}$ protein was the strongest predictor of BPD (unadjusted relative risk $=6.67, p=0.0001$ ) and remained so after adjusting for the other three variables, i.e. birth weight of

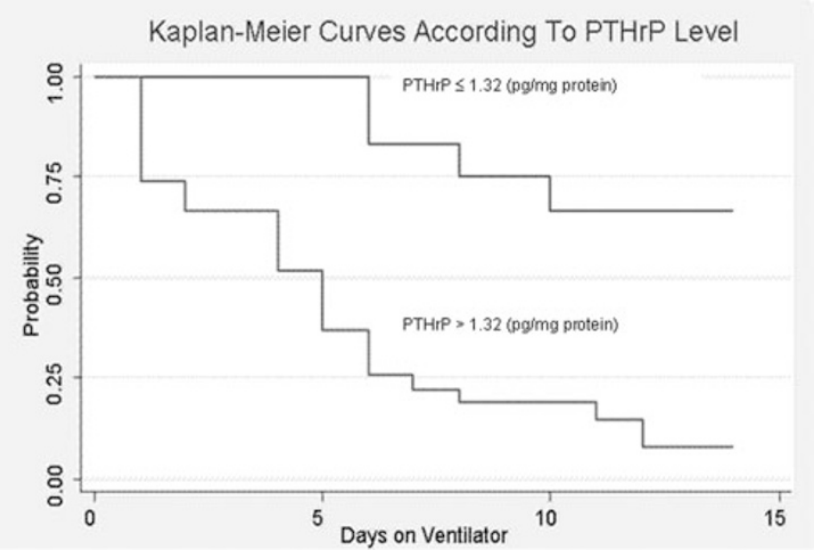

Figure 5. Kaplan-Meier curves for the likelihood of weaning off ventilation based on first week TA PTHrP level. A higher tracheal aspirate PTHrP level $(>1.32 \mathrm{pg} / \mathrm{mg}$ protein) is predicts weaning off ventilator significantly earlier (log-rank test $p<0.0001$ ).

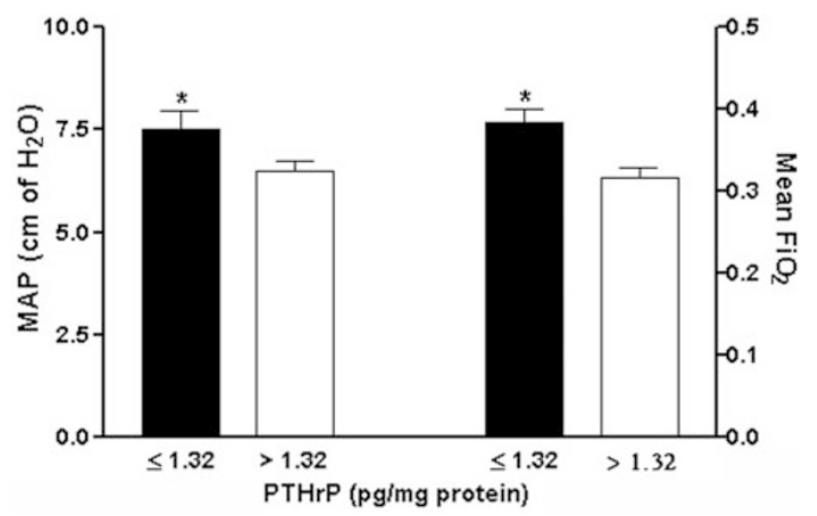

Figure 6. Infants with tracheal aspirate PTHrP values $\leq 1.32 \mathrm{pg} / \mathrm{mg}$ protein required significantly higher mean airway pressure (MAP) and mean fractional inspired oxygen $\left(\mathrm{FiO}_{2}\right)$ concentration than did infants with tracheal aspirate PTHrP values $>1.32 \mathrm{pg} / \mathrm{mg}$ protein. ${ }^{*} p<0.05$.

Table 1. Birth weight, gestational age, and sex distribution in very low birth weight infants with low and high tracheal aspirate PTHrP contents

\begin{tabular}{lccc}
\hline $\begin{array}{c}\text { Tracheal aspirate PTHrP } \\
\text { (pg/mg protein) }\end{array}$ & $\begin{array}{c}\leq 1.32 \\
(n=12)\end{array}$ & $\begin{array}{c}>1.32 \\
(n=28)\end{array}$ & $p$ Value \\
\hline Birth weight (mean \pm SEM) & $838 \pm 74$ & $988 \pm 59$ & 0.155 \\
Gestational age (mean \pm SEM) & $27 \pm 0.7$ & $28 \pm 0.4$ & 0.06 \\
Male gender $(\%)$ & 66 & 46 & $>0.05$ \\
\hline
\end{tabular}

$<1000 \mathrm{~g}, \mathrm{GA}$ of $<28 \mathrm{wk}$, and male gender (adjusted relative risk $=8.73, p=0.001$ ). Furthermore, risks from low birth weight, early GA, and maleness are all attenuated after accounting for low PTHrP (Table 2).

\section{DISCUSSION}

Our data clearly show that VLBWI who later go on to develop BPD have significantly lower TA PTHrP levels during the first week of life than infants who do not develop BPD. A TA PTHrP level of $<1.32 \mathrm{pg} / \mathrm{mg}$ protein is highly predictive for the later onset of BPD (sensitivity $=76.9 \%$ and specificity $=88.5 \%$ ). TA PTHrP levels also inversely correlate with the duration of ventilation needed in VLBWI. Infants who required a longer duration of ventilation ( $2-3$ wk versus 
Table 2. Relative risk of BPD according to tracheal aspirate PTHrP content, birth weight, gestational age, and male gender

\begin{tabular}{|c|c|c|c|c|}
\hline Potential risk factor for BPD & \multicolumn{2}{|c|}{ Unadjusted relative risk of BPD } & \multicolumn{2}{|c|}{ Adjusted* relative risk of BPD } \\
\hline Birth weight $<1000 \mathrm{~g}$ & $44 \% / 14 \%=3.08$ & 0.06 & $0.35 / 0.26=1.31$ & 0.58 \\
\hline Male gender & $35 \% / 28 \%=1.26$ & 0.64 & $0.32 / 0.31=1.01$ & 0.97 \\
\hline PTHrP $\leq 1.32 \mathrm{pg} / \mathrm{mg}$ & $77 \% / 12 \%=6.67$ & 0.0001 & $0.74 / 0.09=8.73$ & 0.001 \\
\hline
\end{tabular}

* Simultaneous mutual adjustment for all other factors in this table. The adjustment for birth weight (BW) is almost identical when alternatively adjusting for PTHrP only ( $\mathrm{RR}=1.31, p=0.57$ ), as is adjustment for PTHrP when adjusting for each of the other three factors individually (RR $=8.68,9.13,9.16 ; p \leq$ $0.001)$.

$\dagger$ Ratio of the percentage of infants developing BPD among those with the risk factor to those without the risk factor.

$\ddagger$ Ratio of the adjusted probability of developing BPD among those with the risk factor to those without the risk factor.

$1 \mathrm{wk}$ ) had significantly lower TA PTHrP levels during the first week of life. Overall, TA PTHrP levels are much more predictive of the later onset of BPD compared with the other known predictors of BPD, such as birth weight $(<1000 \mathrm{~g})$, GA $(<28 w k)$, and male gender. However, in contrast to PTHrP levels, MMP-8 levels do not predict the subsequent development of BPD in VLBWI.

Our observation of lower PTHrP levels in TA in infants with adverse pulmonary outcomes is consistent with the existing data from previous neonatal (17), adult (18), and animal studies (12). Speziale et al. (17) observed significantly lower TA PTHrP levels in infants born at less than $35 \mathrm{wk}$ of gestation versus those born near term; in infants with a $\mathrm{BW}$ of $<2 \mathrm{~kg}$ versus those with $\mathrm{BW}$ of $>2 \mathrm{~kg}$; male preterm $(<35$ wk) infants versus females; and in preterm infants who had severe RDS versus infants with milder RDS. These data reinforce the association of PTHrP levels with indices of lung maturation, and its lower levels correlating with RDS and its risk factors (17). Stern et al. (18) reported that PTHrP levels correlated inversely with the lung injury score, severity of infiltrates on chest radiographs, and TA albumin concentration in adults with acute respiratory distress syndrome. Further, we have previously reported that tracheal PTHrP concentrations remain low after birth in baboons, who, following ventilatorinduced lung injury, go on to develop BPD, versus a 10 -fold rise over the first $10 \mathrm{~d}$ in control animals that did not develop BPD (12).

It is noteworthy that in this study, we did not find male gender and GA $<28$ wk to be independently associated with an increased risk of BPD. This might be a reflection of either a relatively small sample size in this study or the fact that development of BPD is multifactorial and is likely to be determined by a combination of exposure to postnatal risk factors such as volutrauma, hyperoxia, and infection in a susceptible host rather than just by male gender and low birth weight.

PTHrP is a stretch-regulated gene expressed in the lung by the ATII cell. PTHrP determines the key epithelialmesenchymal interactions in the developing lung (19), and has been shown to be necessary for inducing the endodermal and mesodermal phenotypes of the alveolus $(8,9)$. The PTHrP secreted by epithelial ATII cells acts on its receptor on the adjacent AIF, activating a cAMP-dependent protein kinase A pathway (10) that leads to the activation of peroxisome proliferator activated receptor $\gamma(20)$ and its downstream targets, adipocyte differentiation related protein (19) and leptin (21), facilitating triglyceride uptake by AIF (19). The triglycerides taken up by the AIF are then trafficked to the ATII cell for surfactant phospholipid synthesis (22). In fact, for surfactant phospholipid synthesis, ATII cells are dependent upon AIF to recruit neutral lipids from the circulation to be trafficked to the ATII cells for surfactant phospholipid synthesis (23). In addition to its role in surfactant phospholipid synthesis, the lipogenic phenotype of the AIF is critically important in providing cytoprotection against oxidant lung injury (24) as well as in promoting ATII cell proliferation and differentiation (12). We have proposed that the transdifferentiation of AIFto-myofibroblasts under the influence of factors that predispose to BPD is a central event in the pathogenesis of BPD in the newborn, and CLD in the adult $(9,12,25)$, as transdifferentiated AIF (i.e. myofibroblasts) are unable to maintain pulmonary alveolar homeostasis, resulting in failed alveolarization and pulmonary fibrosis (12).

In addition to PTHrP, we elected to examine the MMP-8 content of TA because neutrophil-derived MMP-8 has recently been suggested both as a marker of the severity of RDS, as well as a predictor for the development of BPD in preterm infants (14). MMP-8 is expressed by neutrophils and is stored within specific granules, from where it is released and secreted during inflammatory processes. Activated MMP-8 cleaves substrates including collagen types I, II, III, and aggrecan. In addition, MMP-8 is also expressed and secreted by cytokinestimulated mesenchymal cells. Because in our patients TA MMP-8 levels did not correlate with the development of BPD, we conclude that TA PTHrP levels are a better discriminator of alveolar health and disease. However, the observed differences in MMP-8 levels between our study and the previously reported study that found it to be a predictor of BPD could be due to the methodological differences between the two studies since we measured MMP-8 using ELISA versus the Western analysis used in the study by Cederqvist et al. (14).

\section{CONCLUSIONS}

From the data reported here, we conclude that PTHrP levels in TA fluid of ventilated VLBWI correlates with the duration of ventilation required and the likelihood of developing BPD. In contrast, MMP-8 levels do not correlate with the likelihood of developing BPD. However, our observations need to be tested in a larger multi-institutional study. We speculate that 
PTHrP levels in TA reflect the alveolar epithelial-mesenchymal milieu and predict the state of alveolar homeostasis. Therefore, our observations are likely to hold true in a larger study as well.

Acknowledgments. The authors thank biostatistician Peter Christenson, Ph.D., for help with statistical analysis.

\section{REFERENCES}

1. Christou H, Brodsky D 2005 Lung injury and bronchopulmonary dysplasia in newborn infants. J Intensive Care Med 20:76-87

2. Bancalari E, Claure N, Sosenko IR 2003 Bronchopulmonary dysplasia: changes in pathogenesis, epidemiology and definition. Semin Neonatol 8:63-71

3. Jobe AJ 1999 The new BPD: an arrest of lung development. Pediatr Res 46:641-643

4. Donn SM, Sinha SK 2003 Can mechanical ventilation strategies reduce chronic lung disease? Semin Neonatol 8:441-448

5. Coalson JJ 2003 Pathology of new bronchopulmonary dysplasia. Semin Neonatol 8:73-81

6. Speer CP 2003 Inflammation and bronchopulmonary dysplasia. Semin Neonatol 8:29-38

7. Shannon JM, Hyatt BA 2004 Epithelial-mesenchymal interactions in the developing lung. Annu Rev Physiol 66:625-645

8. Hastings RH 2004 Parathyroid hormone-related protein and lung biology. Respir Physiol Neurobiol 142:95-113

9. Torday JS, Rehan VK 2003 Mechanotransduction determines the structure and function of lung and bone: a theoretical model for the pathophysiology of chronic disease. Cell Biochem Biophys 37:235-246

10. Rubin LP, Kifor O, Hua J, Brown EM, Torday JS 1994 Parathyroid hormone (PTH) and PTH-related protein stimulate surfactant phospholipid synthesis in rat fetal lung, apparently by a mesenchymal-epithelial mechanism. Biochim Biophys Acta 1223:91-100

11. Torday JS, Sanchez-Esteban J, Rubin LP 1998 Paracrine mediators of mechanotransduction in lung development. Am J Med Sci 316:205-208
12. Torday JS, Torres E, Rehan VK 2003 The role of fibroblast transdifferentiation in lung epithelial cell proliferation, differentiation, and repair in vitro. Pediatr Pathol Mol Med 22:189-207

13. Rehan V, Torday J 2003 Hyperoxia augments pulmonary lipofibroblast-tomyofibroblast transdifferentiation. Cell Biochem Biophys 38:239-250

14. Cederqvist K, Sorsa T, Tervahartiala T, Maisi P, Reunanen K, Lassus P, Andersson S 2001 Matrix metalloproteinases-2, -8 , and -9 and TIMP-2 in tracheal aspirates from preterm infants with respiratory distress. Pediatrics 108:686-692

15. Sweet DG, McMahon KJ, Curley AE, O'Connor CM, Halliday HL 2001 Type I collagenases in bronchoalveolar lavage fluid from preterm babies at risk of developing chronic lung disease. Arch Dis Child Fetal Neonatal Ed 84:F168-F171

16. Bradford MM 1976 A rapid and sensitive method for the quantitation of microgram quantities of protein utilizing the principle of protein-dye binding. Anal Biochem $72: 248-254$

17. Speziale MV, Mannino FL, Hastings RH, Deftos LJ 1998 Parathyroid hormonerelated protein $(\mathrm{PTHrP})$ in tracheal aspirates of newborn infants. Pediatr Res 43:660-665

18. Stern JB, Bernard O, Paugam C, Silve C, Mantz J, Aubier M, Crestani B 2002 Parathyroid hormone-related protein in epithelial lining fluid in human negatively correlates with the severity of lung injury. Chest 121:852-857

19. Torday JS, Rehan VK 2002 Stretch-stimulated surfactant synthesis is coordinated by the paracrine actions of PTHrP and leptin. Am J Physiol Lung Cell Mol Physiol 283:L130-L135

20. Rehan VK, Wang Y, Sugano S, Romero S, Chen X, Santos J, Khazanchi A, Torday JS 2005 Mechanism of nicotine-induced pulmonary fibroblast transdifferentiation. Am J Physiol Lung Cell Mol Physiol 289:L667-L676

21. Torday JS, Sun H, Wang L, Torres E, Sunday ME, Rubin LP 2002 Leptin mediates the parathyroid hormone-related protein paracrine stimulation of fetal lung maturation. Am J Physiol Lung Cell Mol Physiol 282:L405-L410

22. Schultz CJ, Torres E, Londos C, Torday JS 2002 Role of adipocyte differentiationrelated protein in surfactant phospholipid synthesis by type II cells. Am J Physiol Lung Cell Mol Physiol 283:L288-L296

23. Torday J, Hua J, Slavin R 1995 Metabolism and fate of neutral lipids of fetal lung fibroblast origin. Biochim Biophys Acta 1254:198-206

24. Torday JS, Torday DP, Gutnick J, Qin J, Rehan V 2001 Biologic role of fetal lung fibroblast triglycerides as antioxidants. Pediatr Res 49:843-849

25. Pardo A, Selman M 2002 Idiopathic pulmonary fibrosis: new insights in its pathogenesis. Int J Biochem Cell Biol 34:1534-1538 